\title{
Four point probe structures with buried electrodes for the electrical characterization of ultrathin conducting films
}

\author{
A.W. Groenland ${ }^{1}$, R.A.M. Wolters ${ }^{1,2}$, A.Y. Kovalgin ${ }^{1} \&$ J. Schmitz ${ }^{1}$ \\ ${ }^{1}$ MESA+ Institute for Nanotechnology, Chair of Semiconductor Components \\ University of Twente, P.O. Box 217, 7500 AE Enschede, The Netherlands. \\ Tel +31 (0)53489 2645, Fax +31(0)534891034 \\ Email: A.W.Groenland@utwente.nl
}

${ }^{2}$ NXP-TSMC Research Center, High Tech Campus 4, 5656 AA Eindhoven, The Netherlands

\begin{abstract}
Test structures for the electrical characterization of ultrathin conductive (ALD) films are presented based on buried electrodes on which the ultrathin film is deposited. This work includes test structure design and fabrication, and the electrical characterization of ALD TiN films down to $4 \mathrm{~nm}$. It is shown that these structures can be used successfully to characterize sub $10 \mathrm{~nm}$ films.
\end{abstract}

\section{INTRODUCTION}

Conducting thin films (sub $10 \mathrm{~nm}$ ) have interesting applications in the field of diffusion barriers for copper metallization and phase change memories [1-4]. Electrical properties can be measured using the four probe method $[5,6]$. For these thin films, van der Pauw structures (vdPs) and Greek Crosses (GCs) are commonly used [7]. However, making electrical contacts to these thin films, using planar technology, is extremely difficult; e.g. etching a via for the contact to such a film requires a very high selectivity. In practice, this is not possible. Enderling and co-workers proposed to use suspended Greek Crosses to overcome this problem. However, this is only suitable for films with a poor step coverage (e.g. deposited via physical vapour deposition (PVD)) [8].

In this work special test structures are presented to measure the electrical properties of thin films in a controlled way. Electrodes are fabricated, which are buried in a planarized dielectric film. A thin film is deposited on top of the electrodes by atomic layer deposition (ALD). The thin film is characterized using the predefined electrode structures, such as the aforementioned vdPs and GCs. The work presented here includes test structure design and fabrication. It is demonstrated that these structures can be used successfully to characterize $7 \mathrm{~nm}$ and $4 \mathrm{~nm}$ thick ALD TiN films.

\section{TEST STRUCTURE FABRICATION}

Square structures are etched in a layer of $0.5 \mu \mathrm{m}$ thermally grown $\mathrm{SiO}_{2}$ on top of a standard silicon wafer using wet chemical etching (Fig. 1a). After an additional oxidation step to insulate the silicon substrate, a layer of $70 \mathrm{~nm}$ TiW is sputtered and patterned to make the electrodes and connections (Fig. 1b). Subsequently a layer of $1 \mu \mathrm{m}$ PECVD $\mathrm{SiO}_{2}$ is deposited (Fig. 1b) and the structure is planarized using chemical mechanical polishing (CMP) (Fig. 1c). On this surface the thin ALD TiN film is deposited which is passivated by in situ ALD $\mathrm{Al}_{2} \mathrm{O}_{3}$ and ex situ PECVD $\mathrm{SiO}_{2}$ layers (Fig. 1d). After patterning the ALD TiN and passivation layers (Fig. 1e), vias are etched towards the buried electrode connections and filled with aluminium to become realize contact pads (Fig. 1f). In this process, structures such as vdPs, GCs, collinear probes and contact strings have been realized. The schematic design and exact device dimensions of a $\mathrm{vdP}$ and GC are shown in Fig. 2. An example of a realized van der Pauw structure is shown in Fig. 3.
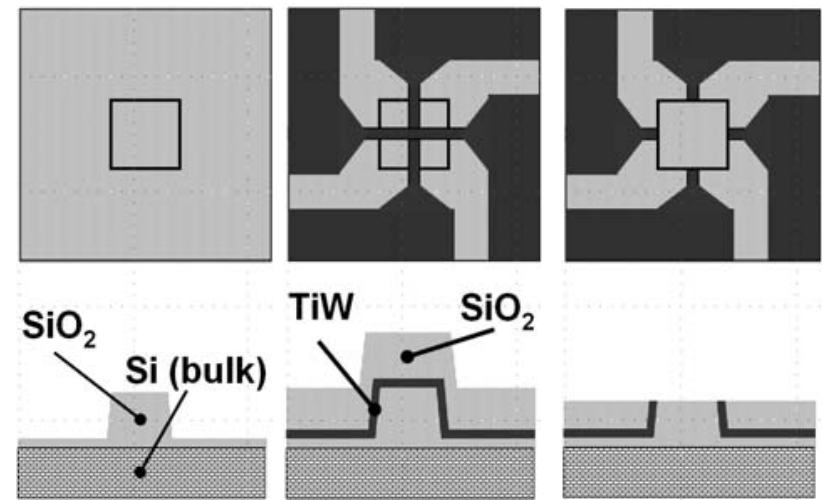

(a)

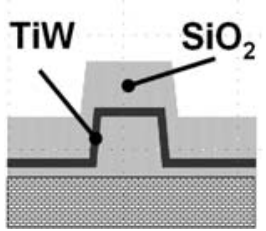

(b)

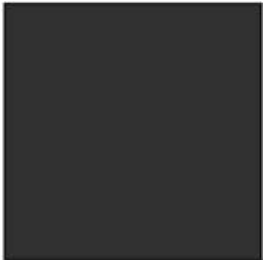

ALD TiN +

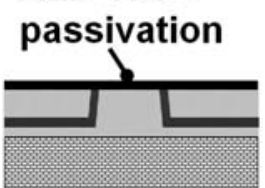

(d)
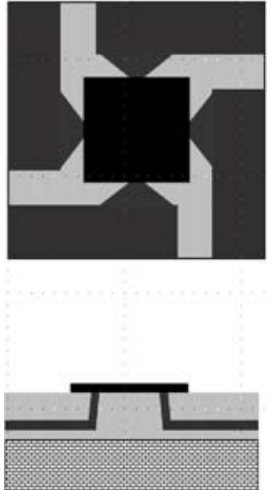

(e)
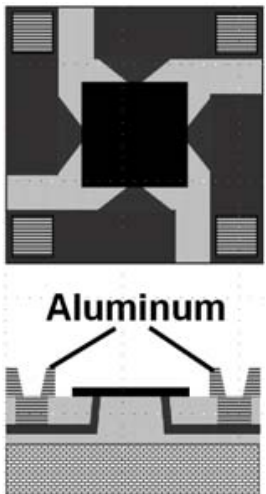

(f)

Fig. 1. Fabrication scheme for a van der Pauw structure (top view (top) and cross-section (bottom)). 


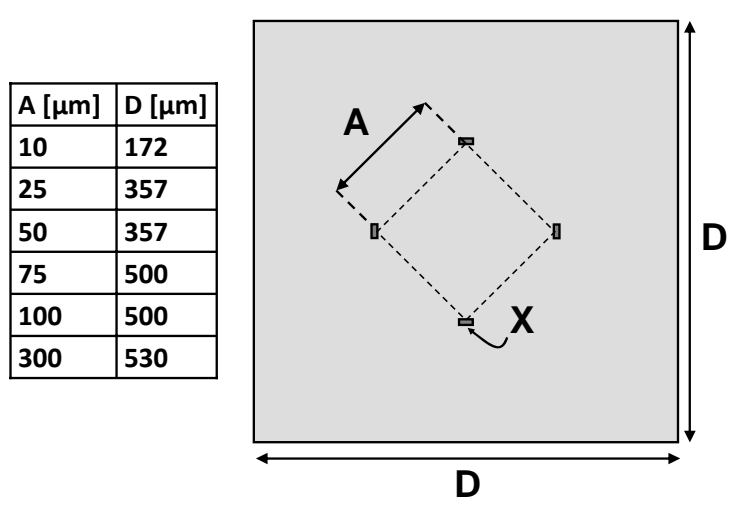

(a)

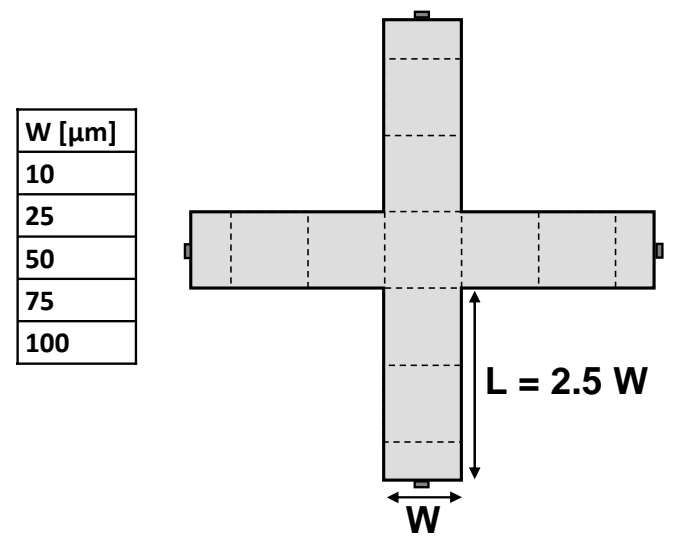

(b)

Fig. 2. Schematic design of a van der Pauw (a) and Greek Cross (b) device. The four contacts of the van der Pauw are situated at the corners of an imaginary square at a distance $A$ that is centred with respect to the ALD film. The ALD film is patterned as a square with dimension $D . X$ indicates the area of a single contact $(2.5 \mu \mathrm{m}$ $\times 0.1 \mu \mathrm{m}$ for all devices). The Greek Cross is designed with the arm length $L$ equal to 2.5 times the width $W$. This reduces the error in the extracted sheet resistance to $<1 \%$ while maintaining maximum sensitivity and minimal Joule heating in the arms of the cross [911]. Dashed lines indicate the (imaginary) squares.
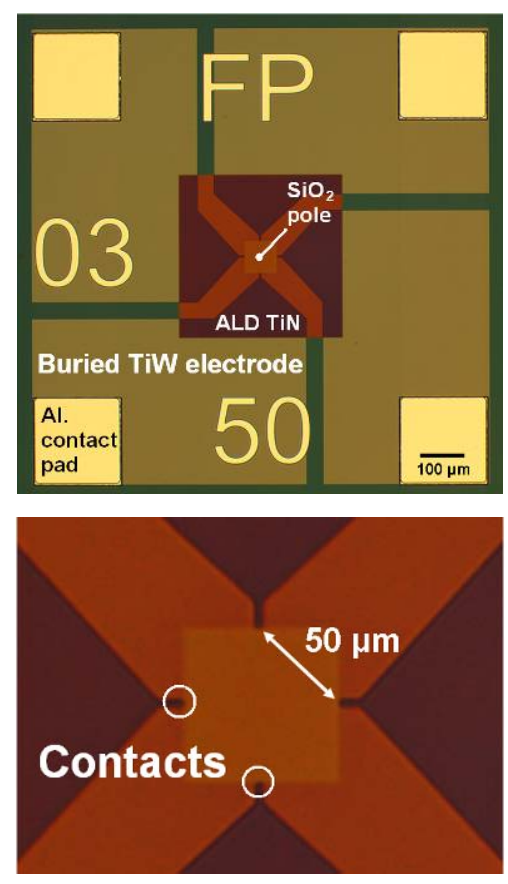

Fig. 3. Optical micrograph of a van der Pauw structure with a probe-to-probe distance of $50 \mu \mathrm{m}$. Whole structure (top) and close up of the electrodes (bottom).

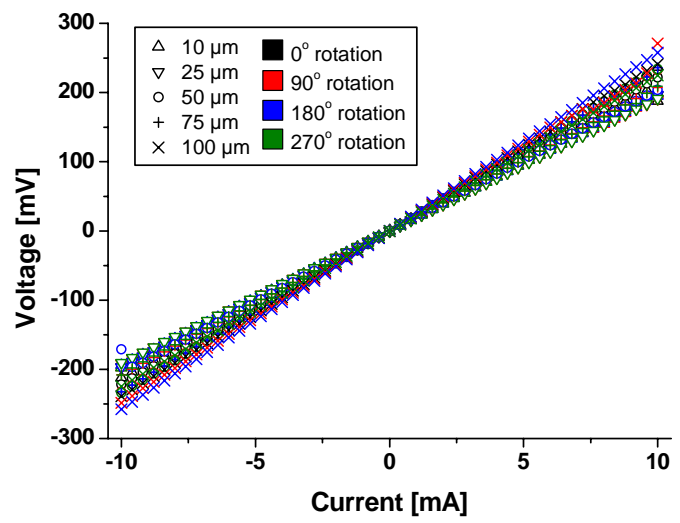

(a)

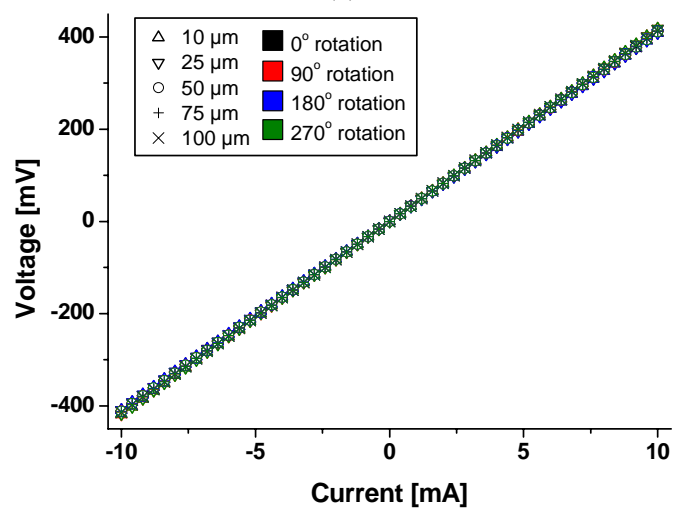

(b)

Fig. 4. Measured $V_{\mathrm{m}}$ versus $I_{\mathrm{m}}$ curves for van der Pauw (a) and Greek Cross (b) test structures for $7 \mathrm{~nm}$ ALD TiN. Dimensions refer to the probe-to-probe distance (van der Pauw) and the size of the central square of the Greek Cross.

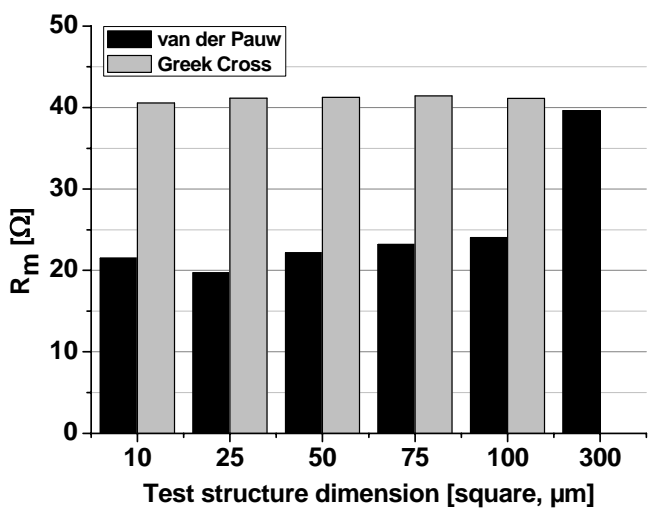

(a)

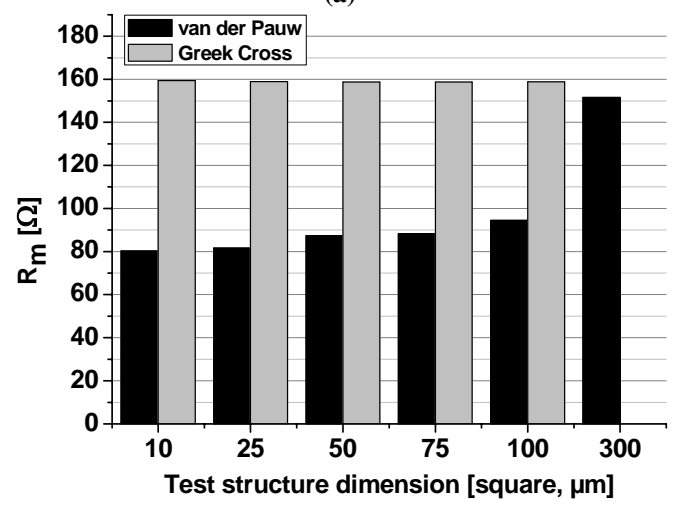

(b)

Fig. 5. Extracted resistances $\left(V_{\mathrm{m}} / I_{\mathrm{m}}\right)$ from van der Pauw and Greek Cross structures for $7 \mathrm{~nm}$ (a) and $4 \mathrm{~nm}$ (b) ALD TiN layers. Resistances are an average of the 4 measured orientations (see Fig.
4). 


\section{EXPERIMENTAL}

The layer thickness of the ALD TiN films is determined using a Woollam M2000 Spectroscopic Ellipsometer (SE) in the energy range 0.7-5 eV. Measurements were taken in situ directly after deposition. From the recorded SE data, the ALD TiN layer thickness is derived using a model containing the optical constants of all sub-layers. The ALD TiN layer thickness and the contact area of the electrodes are verified by HRSEM on cross-sections of the sample.

For the electrical characterization, $I V$-measurements were carried out using a HP4156B or Keithley 4200 precision semiconductor parameter analyser in combination with a Cascade Microtech probe station. For the measurements at elevated temperatures, the temperature controlled chuck of the probe station was used.

\section{RESULTS}

\section{A. Measurements at room temperature}

For a $7 \mathrm{~nm}$ ALD TiN layer, $I V$-curves are measured from van der Pauw (Fig. 4a) and Greek Cross structures (Fig. 4b) having probe-to-probe distances (vdP) or central squares (GC) in the range of $10 \times 10$ to $100 \times 100 \mu \mathrm{m}^{2}$. All devices are measured over all 4 orientations. From (solely) the slope of both sets of $I V$-curves, the ('measured') resistance $\left(R_{\mathrm{m}}=V_{\mathrm{m}} / I_{\mathrm{m}}\right)$ is calculated and averaged over the 4 orientations. The results are shown in Fig. 5a. In Fig. $5 \mathrm{~b} R_{\mathrm{m}}$ values are shown for a $4 \mathrm{~nm}$ ALD TiN layer. Values of $R_{\mathrm{m}}$ values extracted from a van der Pauw device with a probe-to-probe distance of $300 \mu \mathrm{m}$ are included in the graphs

For van der Pauw structures, a relatively large spread in the $I V$-characteristics (Fig. 4a) is observed for different device dimensions and also between different orientations of a single device. For Greek Crosses (Fig. $4 \mathrm{~b})$ this is not the case.

From Fig. 5 it is observed that resistances extracted from van der Pauw structures (except for the $300 \mu \mathrm{m}$ device) are significantly lower than those extracted from Greek

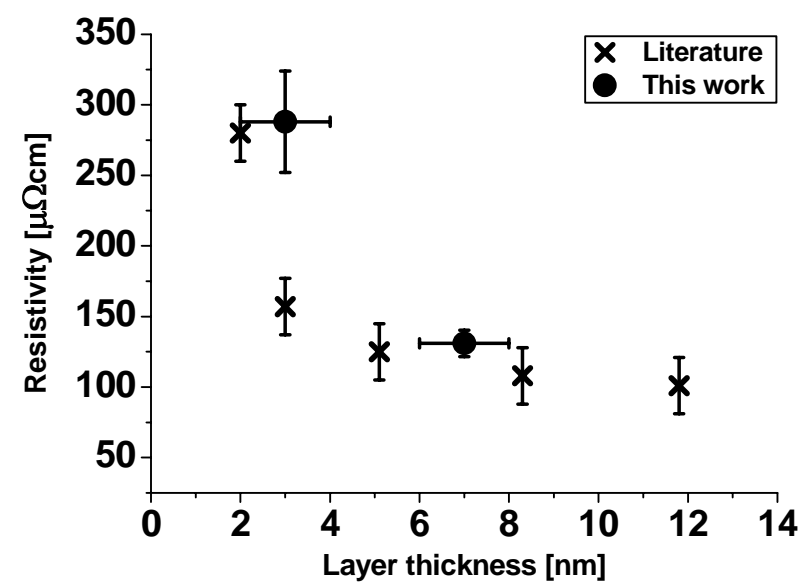

Fig. 6. Resistivity versus layer thickness for ALD TiN films. Literature values are reprinted from [12]. The uncertainty in our data is due to uncertainties in the layer thickness, as derived from spectroscopic ellipsometer (SE) measurements.
Crosses. A slight increase in $R_{\mathrm{m}}$ is observed for larger devices. The reduced measured resistance may be related to the finite contact area $\left(\sim 2,5 \times 0.1 \mu^{2}\right)$ of the electrodes (i.e. not point-like contacts) in the van der Pauw structures $[5,13,14]$.

Furthermore, an increase is observed in $R_{\mathrm{m}}$ extracted from van der Pauw structures for larger devices. This is most likely due to the fact that the edge of the ALD layer is situated further away from the electrodes for smaller devices, thereby violating one of van der Pauw's boundary conditions that the contacts should be at the circumference of the sample [5]. This is supported by measurements on the $300 \times 300 \mu \mathrm{m}^{2}$ van der Pauw device in which the electrodes are close to the ALD layer edge: they yield virtually the same values for $R_{\mathrm{m}}$ as extracted from Greek Crosses.

For Greek Cross devices, the sheet resistance $\left(R_{\square}\right)$ can be calculated from $R_{\mathrm{m}}$ using a correction factor of $\pi / \ln (2)$ [7]. The resistivity $(\rho)$ is calculated from $R_{\square}$ and the layer thickness (averaged over all 5 devices). Values of 131 and $288 \mu \Omega \mathrm{cm}$ are obtained for 7 and $4 \mathrm{~nm}$ ALD TiN respectively. These results are shown in Fig. 6 together with results from literature [12]. The error in $\rho$ originates from uncertainty in the ALD TiN layer thickness. Our extracted resistivity values are slightly higher than the literature values, but still realistic $[2,12,15]$. The difference is may be due to differences in material composition and/or the uncertainty in the layer thickness.

For van der Pauw devices it is more difficult to extract $R_{\square}$. As mentioned above, the contacts have finite size and are not placed at the circumference of the sample, so a correction factor larger than the standard correction factor of $\pi / \ln (2)$ has to be used. This factor cannot be derived easily in an analytical way, but might be obtained from finite element modelling [16]. This is beyond the scope of our present work.

\section{B. Measurements at elevated temperatures}

Both van der Pauw and Greek Cross devices can be used for measurements at different temperatures to find the temperature coefficient of resistance (TCR) of the ALD TiN film. For a $7 \mathrm{~nm}$ ALD TiN film, resistance measurements in the range of $25-175{ }^{\circ} \mathrm{C}$ are shown in Fig. 7. In Fig. 7a the measured resistance $R_{\mathrm{m}}$ of a $75 \mu \mathrm{m}$ van der Pauw and a $75 \mu \mathrm{m}$ Greek Cross device are shown. A linear fit through the data can be described as:

$$
R_{m}=a T+R_{0}
$$

with $R_{\mathrm{m}}$ the $V_{\mathrm{m}} / I_{\mathrm{m}}[\Omega]$ and $T$ the temperature $\left[{ }^{\circ} \mathrm{C}\right]$. The temperature coefficient of resistance $\left(\beta\right.$ or TCR) $\left[{ }^{\circ} \mathrm{C}\right]$ can be extracted from the fit parameters $a$ and $R_{0}$ by rewriting (1):

$$
R_{m}=R_{0}(1+\beta T) \rightarrow \frac{R_{m}}{R_{0}}=\beta T+1
$$

with $\beta=a / R_{0}[17]$. 


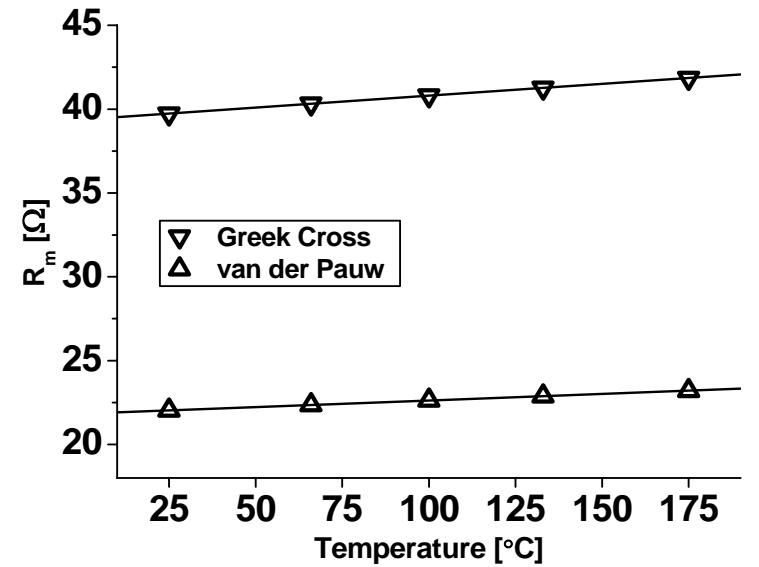

(a)

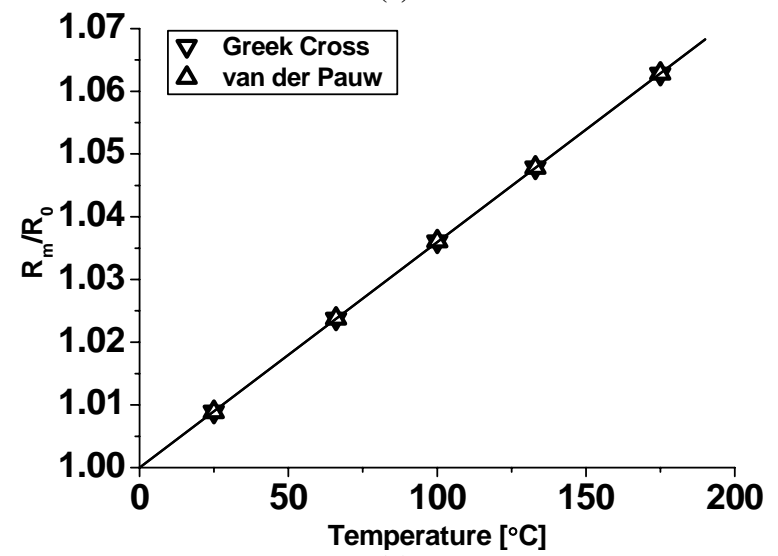

(b)

Fig. 7. Resistance versus temperature curves of $75 \mu \mathrm{m}$ van der Pauw and $75 \mu \mathrm{m}$ Greek Cross devices for a $7 \mathrm{~nm}$ ALD TiN layer. (a) the measured resistance $\left(R_{\mathrm{m}}\right)$ and (b) $R_{\mathrm{m}}$ normalized on the (extrapolated) $R_{\mathrm{m}}$ value at $0{ }^{\circ} \mathrm{C}\left(R_{0}\right)$. Lines are linear fits through the data. The temperature coefficient of resistance (TCR) is extracted from the slope of (b) (see text).

In Fig. 7b $R_{\mathrm{m}} / R_{0}$ is plotted versus temperature for the same devices as in Fig. 7a. Although the absolute $R_{\mathrm{m}}$ values of the van der Pauw and the Greek Cross differ, it is observed that the normalized resistance values yield the same TCR $\left(3.59 \times 10^{-4} /{ }^{\circ} \mathrm{C}\right)$ for both devices.

In Fig. 8 TCR values for van der Pauw and Greek Cross devices with dimensions in the range of 10-300 $\mu \mathrm{m}$ are shown. It is observed that the spread in TCR values obtained from Greek Crosses is very low (within $\sim 1 \%$ of their average value). For van der Pauw devices a larger spread (7\% at maximum) in TCR values is observed. This spread might be related to a spread in device properties across the wafer; the 75 and $100 \mu \mathrm{m} \mathrm{vdP} \mathrm{\&}$ GC devices were positioned close to each other (neighbouring devices) and yield virtually equal TCR values (within $0.8 \%$ of their average value). All other devices were measured at different locations across the wafer (i.e. not within the same die). It appears van der Pauw devices are more sensitive to this spread in device properties than Greek Crosses.

The extracted TCR from Greek Cross devices of $3.5 \times$ $10^{-4} /{ }^{\circ} \mathrm{C}$ is roughly $40 \%$ lower than the literature value of $5.5 \times 10^{-4} /{ }^{\circ} \mathrm{C}$, measured by Langereis and co-workers on an ALD TiN layer that is deposited under similar

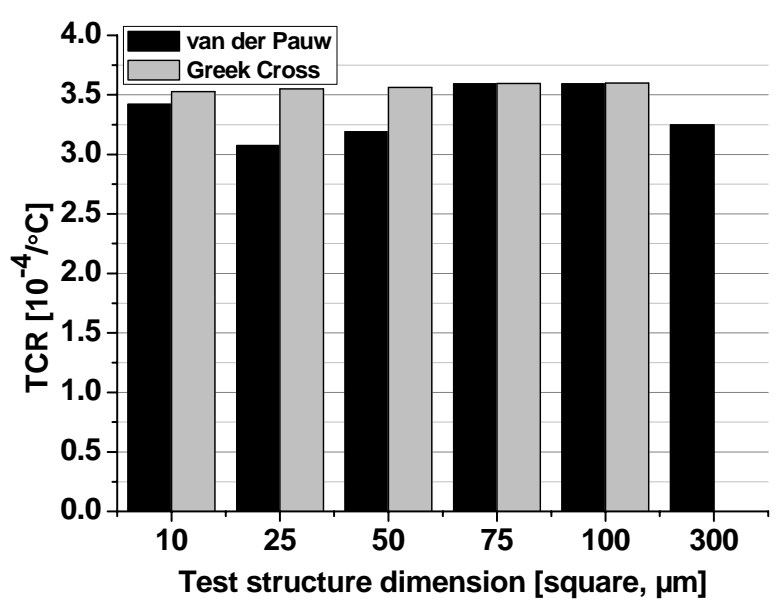

Fig. 8. Temperature coefficient of resistance (TCR) values of a $7 \mathrm{~nm}$ ALD TiN film for various van der Pauw and Greek Cross devices. Dimensions refer to the probe-to-probe distance (van der Pauw) and the size of the central square of the Greek Cross.

conditions $[12,18]$. The difference is most likely due to a reduction of the TCR as a result of enhanced surface scattering which occurs for extremely thin films. Langereis and co-workers used a $33 \mathrm{~nm}$ thick film for the determination of their TCR which is significantly thicker than our $7 \mathrm{~nm}$ film $[12,18]$.

\section{CONCLUSIONS}

Test structures for the electrical characterization of ultrathin conductive films are presented based on buried electrodes on which the ultrathin film is deposited. Electrical measurements on Greek Cross structures yield resistance values which are independent of the device dimensions $\left(10 \times 10\right.$ to $\left.100 \times 100 \mu \mathrm{m}^{2}\right)$ and the extracted values for the resistivity of 288 and $133 \mu \Omega \mathrm{cm}$ for 4 and $7 \mathrm{~nm}$ ALD TiN layers, respectively, are realistic. Both van der Pauw and Greek Cross devices can be used for the extraction of the temperature coefficient of resistance (TCR) of ALD TiN. For a $7 \mathrm{~nm}$ ALD TiN layer, a TCR value of $3.5 \times 10^{-4} /{ }^{\circ} \mathrm{C}$ is found.

\section{ACKNOWLEDGMENT}

The authors thank M. Hoekman (LioniX BV) for help with CMP processing, T. Aarnink (MESA+) for help with the ALD of TiN and the Dutch Technology Foundation STW for financial support of this project (07682).

\section{REFERENCES}

International Technology Roadmap for Semiconductors: 2007 Update, International Sematech, 2007. Available from: http://public.itrs.net.

[2] Ch. 2: Atomic layer deposition, in Handbook of thin film materials part 1: Deposition and processing of thin films (2002), vol. 1, edited by H. S. Nalwa, Academic Press. 
[3] D. T. Castro, L. Goux, G. A. M. Hurkx, K. Attenborough, R. Delhougne, J. Lisoni, F. J. Jedema, M. A. A. t Zandt, R. A. M. Wolters, D. J. Gravesteijn, M. Verheijen, M. Kaiser, and R. G. R. Weemaes, "Evidence of the Thermo-Electric Thomson Effect and Influence on the Program Conditions and Cell Optimization in PhaseChange Memory Cells," in 2007 IEEE Electron Devices Meeting, pp 315-318.

[4] J. Sarkar and B. Gleixner, Applied Physics Letters, Vol. 91, No. 23, 2007, pp. 233506-3.

[5] L. van der Pauw, Philips Research Reports, Vol. 13, No. 1, 1958, pp. 1-9.

[6] L. van der Pauw, Philips Technical Review, Vol. 20, No., 1958, pp. 220-224.

[7] M. G. Buehler and W. R. Thurber, Journal of The Electrochemical Society, Vol. 125, No. 4, 1978, pp. 645-650.

[8] S. Enderling, C. L. Brown, III, S. Smith, M. H. Dicks, J. T. M. Stevenson, M. Mitkova, M. N. Kozicki, and A. J. Walton, Semiconductor Manufacturing, IEEE Transactions on, Vol. 19, No. 1, 2006, pp. 2-9.

[9] S. Enderling, M. H. Dicks, S. Smith, J. T. M. Stevenson, and A. J. Walton, "Thermal design considerations for Greek cross test structures," in 2003 IEEE International Conference on Microelectronic Test Structures, pp 8-13.

[10] S. Smith, "Sheet Resistance and Electrical Linewidth Test Structures for Semiconductor Process Characterisation" (2003), Ph.D. thesis, University of Edinburgh, Scotland.
[11] Y. Sun, J. Shi, and Q. Meng, Semiconductor Science and Technology, Vol. 11, No. 5, 1996, pp. 805-811.

[12] E. Langereis, S. B. S. Heil, M. C. M. van de Sanden, and W. M. M. Kessels, Journal of Applied Physics, Vol. 100, No. 2, 2006, pp. 023534.

[13] W. Versnel, Solid-State Electronics, Vol. 21, No. 10, 1978, pp. 1261-1268.

[14] M. Cornils, M. Doelle, and O. Paul, IEEE Transactions on Electron Devices, Vol. 54, No. 10, 2007, pp. 2756-2761.

[15] S. B. S. Heil, E. Langereis, F. Roozeboom, M. C. M. van de Sanden, and W. M. M. Kessels, Journal of the Electrochemical Society, Vol. 153, No. 11, 2006, pp. G956-G965.

[16] N. M. P. Guillaume, M. W. Cresswell, R. A. Allen, S. Everist, and L. W. Linholm, "Comparison of sheet-resistance measurements obtained by standard and small-area four-point probing," in 1999 IEEE International Conference on Microelectronic Test Structures, pp 62-66.

[17] CRC Handbook of Chemistry and Physics (1987), ed, edited by CRC Press, Inc., 2000 Corporate Blvd., N.W., Boca Raton, Florida, 33431, USA.

[18] E. Langereis, private communication, 2009. 